\title{
现场模拟光照条件对南极大磷虾 冬季耗氧率的影响研究
}

\author{
胡光照 ${ }^{1,2}$, 刘子俊 ${ }^{1,2}$, 朱国平 ${ }^{1,2,3,4}$ \\ (1. 上海海洋大学海洋科学学院, 上海 201306 ; 2. 上海海洋大学极地研究中心, 上海 201306; \\ 3. 大洋渔业资源可持续开发教育部重点实验室极地海洋生态系统研究室, 上海 201306; \\ 4. 国家远洋渔业工程技术研究中心，上海 210306)
}

\begin{abstract}
摘 要: 为探究海上现场光照条件对冬季南极大磷虾 (Euphausia superba) 代谢的影响, 基于海上暂养实验, 采 用黑暗组和光照组两种不同的实验条件,对 9 尾南极大磷虾个体进行了连续 $11 \mathrm{~d}$ 的耗氧率研究。结果表明, 光照条件下南极大磷虾的单位体质量耗氧率平均为 $(0.2704 \pm 0.0740) \mu \mathrm{L} \cdot(\mathrm{mg} \cdot \mathrm{h})^{-1}$, 黑暗条件下的单位 体质量耗氧率平均为 $(0.2673 \pm 0.0840) \mu \mathrm{L} \cdot(\mathrm{mg} \cdot \mathrm{h})^{-1}$ 。在光照与黑暗条件下南极大磷虾呼吸率无显著 差异。通过对比发现, 冬季南极大磷虾的耗氧率明显低于夏季的耗氧率 $\left[0.63 \sim 0.88 \mu \mathrm{L} \cdot(\mathrm{mg} \cdot \mathrm{h})^{-1}\right]$, 且改 变一个自然日的光照条件对南极大磷虾耗氧率无显著影响, 但会在一定程度上影响南极大磷虾呼吸率的稳 定性。研究验证了冬季南极大磷虾处于一种低代谢模式状况, 并证明了该模式的稳定性。相关结果可为揭示 南极大磷虾越冬机制提供基础数据。
\end{abstract}

关键词: 南极大磷虾; 耗氧率; 越冬; 新陈代谢

中图分类号: S 968 文献标志码: A

南极大磷虾 (Euphausia superba, 以下简称磷 虾) 为磷虾目大型海洋甲壳类浮游动物。该种在 南大洋生态系统中起核心作用, 被认为是南大洋 生态系统的关键物种, 是连接初级生产者和脊椎 动物捕食者之间的主要生物 ${ }^{[1]}$ 。在南大洋大部 分地区被冰覆盖、水体中浮游植物含量极低的冬 季, 磷虾如何生存是南大洋海洋生态系统动力学 计划 (SO GLOBEC) 研究的关键问题之一 ${ }^{[2]}$, 也一 直是全球极地生态学家极为感兴趣的重要科学 问题。总的来讲,成体磷虾的越冬机制通常可分 为两类:一类是非捕食机制,包括使用体内已储 存的脂质 ${ }^{[3]}$, 降低体内的代谢率 ${ }^{[4-6]}$ 及逆生长 ${ }^{[7]}$ 等; 另一类则是改变捕食策略以及转换食物来 源, 即由夏季的主要摄食对象 (如硅藻等浮游植 物) 转变为冰藻以及海冰底下的小型浮游动物
等 $^{[8-9]}$ 和海底碎屑 ${ }^{[4]}$ 。其中, 降低代谢率被认为 是成体磷虾最有效的冬季节能机制之一 ${ }^{[5,9]}$ 。与 夏季相比, 冬季生物体内代谢关键酶一一柠檬酸 合成酶 (CS) 的活性显著降低, 这也导致磷虾的 $\mathrm{CS}$ 活性和核糖核酸: 脱氧核糖核酸( RNA：DNA) 比值出现明显的季节性变化 ${ }^{[10]}$ 。代谢率的降低 可能是冬季磷虾的主要生理反应,但导致这种降 低的机制仍不清楚。有报道 ${ }^{[11]}$ 认为,光周期的变 化是磷虾代谢率季节性变化的主要诱因之一。

为探究光周期对磷虾代谢周期的影响,一些 学者通过不同方法进行了研究。TESCHKE 等 ${ }^{[11]}$ 通过陆基暂养实验发现, 光照强度会使磷虾的耗 氧率升高并提升代谢酶苹果酸脱氢酶 $(\mathrm{MDH})$ 的 活性, 验证了环境光照状态的变化对磷虾的生理 参数 (如摄食和代谢率) 变化具有重要影响。

收稿日期: $2020-07-21$

基金项目：国家自然科学基金项目(41776185);国家重点研发计划项目(2018YFC1406801)

作者简介：胡光照(1994一), 男,硕士研究生, 主要从事海洋生物学研究。E-mail:hugz123@ foxmail.com

通信作者: 朱国平, 教授。E-mail:gpzhu@ shou. edu.cn 
BROWN 等 ${ }^{[12]}$ 的实验却得出了相反的结论, 其结 果显示, 黑暗条件下的磷虾耗氧率高于处于光照 条件下的磷虾, 可能是由于黑暗条件下磷虾更活 跃, 游泳行为增多, 由此使得耗氧率增加。上述 研究均说明了光照条件会影响磷虾的行为和生 理。总的来讲,有关冬季磷虾生理的现场研究仍 较为有限。我国学者通过现场实验对磷虾的行 为和耐受性等进行了研究 ${ }^{[13-16]}$, 并分析了光照 条件对磷虾垂直分布的影响 ${ }^{[17-19]}$, 但尚未有研 究涉及到生理。因此,通过现场实验了解光照变 化对磷虾生理的影响很有必要。有氧代谢时释 放的热量与消耗的氧量呈正比, 因此在海洋生物 生理代谢研究中, 耗氧率可直接作为衡量代谢率 高低的一个指标 ${ }^{[20]}$ 。鉴于此,本研究通过海上现 场暂养实验就不同光照条件对磷虾代谢和生理 状态的影响进行分析, 以期了解冬季磷虾代谢率 对光照条件变化产生的反馈, 为揭示磷虾越冬机 制提供基础数据。

\section{1 材料与方法}

\section{1 实验设置}

实验所用磷虾活体由大型拖网渔船 “龙腾” 从事渔业生产过程中取得,采集时间为 2019 年 6 月 20-22 日, 取样地点为南设德兰群岛水域 $\left(62^{\circ} 33^{\prime} \mathrm{S} 、 59^{\circ} 28^{\prime} \mathrm{W}\right)$, 采样海域海水温度为 $-1.8{ }^{\circ} \mathrm{C}$, 水深为 $800 \sim 1000 \mathrm{~m}$, 虾群深度为 $50 \sim$ $140 \mathrm{~m}$, 空气温度为 $-11{ }^{\circ} \mathrm{C}$ 。采集到的活体磷虾 暂养于 $100 \mathrm{~L}$ 的水槽中, 暂养水体为现场采集的 自然海水 (水温为 $-1.2{ }^{\circ} \mathrm{C} \sim 0.5{ }^{\circ} \mathrm{C}$ )。暂养箱放 置于平均室温为 $0{ }^{\circ} \mathrm{C}$ 的隔离空间内, 光照强度为 $90 \sim 150 \mathrm{~lx}$ (与所在海域自然光照强度基本相符) (图 1)。暂养期间 (4 6 d) 未投饵料, 暂养箱每 $12 \mathrm{~h}$ 换水一次, 每次换水 $4 / 5$, 并将箱底的死亡个 体、虾壳和粪便等由底部排水口排出。至 6 月 26 日, 水箱内每日死亡个体的数量趋于稳定 (5 尾), 剩余磷虾个体状态保持良好, 随机选取活性较好 的 9 尾磷虾进行光照对比实验。

\section{2 实验过程}

实验开始于 2019 年 6 月 26 日 23:00。将 9 尾磷虾分成 2 组, A 组 5 尾磷虾, B 组 4 尾磷虾 (在实验进行到第 3 天时, 磷虾编号 2 死亡, 故加 人样本 9) (表 1 )。所有实验个体分别置于容积 为 $630 \mathrm{~mL}$ 并注人当地海水的白色塑料瓶内后封 口。封口后的实验塑料瓶放人装有当地循环海 水的 $100 \mathrm{~L}$ 箱体内, 使得实验个体所处的温度始 终与当地海水保持一致。实验期间,使用温盐深 仪 (CTD, 型号为 RBR) 对每次实验的海水温度和 盐度进行记录, 海水盐度在 $32.878 \sim 32.968$ 之间,

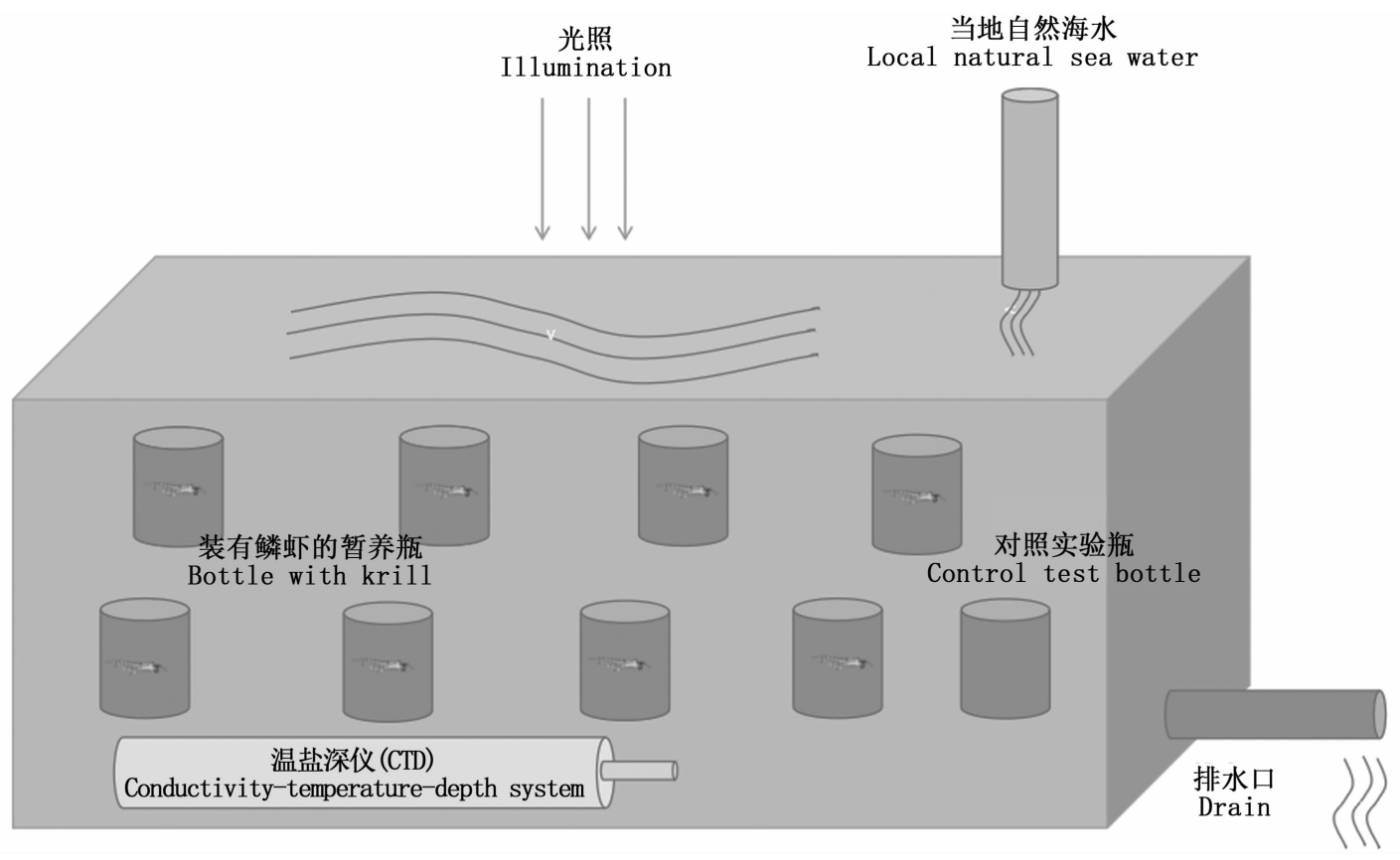

图 1 南极大磷虾暂养装置示意图

Fig. 1 Schematic map of aquarium tank for Euphausia superba 
表 1 实验南极大磷虾样本基本信息

Tab. 1 Basic information about Euphausia superba specimens used in this study

\begin{tabular}{ccccc}
\hline $\begin{array}{c}\text { 组别 } \\
\text { Group }\end{array}$ & $\begin{array}{c}\text { 磷虾编号 } \\
\text { Krill serial }\end{array}$ & $\begin{array}{c}\text { 体长 } / \mathrm{cm} \\
\text { Body length }\end{array}$ & $\begin{array}{c}\text { 干重 } / \mathrm{mg} \\
\text { Dry weight }\end{array}$ & $\begin{array}{c}\text { 性成熟度 } \\
\text { Sexual maturity }\end{array}$ \\
\hline A & 1 & 43.21 & 119.1 & $2 \mathrm{CF}$ \\
A & 2 & 43.68 & 194.7 & $2 \mathrm{DF}$ \\
A & 3 & 40.33 & 157.2 & $2 \mathrm{CF}$ \\
A & 4 & 41.30 & 63.7 & $2 \mathrm{CF}$ \\
A & 9 & 44.65 & 82.6 & $2 \mathrm{CM}$ \\
B & 5 & 41.89 & 97.4 & $2 \mathrm{CF}$ \\
B & 6 & 38.21 & 137.2 & $2 \mathrm{CF}$ \\
B & 7 & 47.56 & 117.2 & $2 \mathrm{CM}$ \\
B & 8 & 44.25 & & $2 \mathrm{CM}$ \\
\hline
\end{tabular}

注:性成熟度参考 MAKAROV 和 DENYS ${ }^{[21]}$ 与 BARGMANN ${ }^{[22]}$ 文献标准进行判定, 雌性未成体分为 $2 \mathrm{BF} 、 2 \mathrm{CF} 、 2 \mathrm{DF} 3$ 期, 雄性未成体分为 $2 \mathrm{AM} 、 2 \mathrm{BM} 、 2 \mathrm{CM} 3$ 期

Note: Sexual maturity is determined by classification protocols provided by MAKAROV and DENYS ${ }^{[21]}$ and BARGMANN ${ }^{[22]}$, female sub-adults are divided into three stages, $2 \mathrm{BF}, 2 \mathrm{CF}$, and $2 \mathrm{DF}$, and male sub-adults are divided into three stages, $2 \mathrm{AM}, 2 \mathrm{BM}$, and $2 \mathrm{CM}$

较为稳定。使用 TES-1322A 型电子照度计测量 实验场地光照强度, 并对实验期间的照度进行调 控, 以保持光照强度尽量恒定, 暂养水箱水体表 面光照强度约为 $100 \mathrm{~lx}$ 。除光照条件外, 实验期 间的其他各项环境数据均相同。直至实验完成, 剩余所有磷虾个体均保持较好活性。

针对 A 组个体, 在光照条件下放置约 $24 \mathrm{~h}$ 后,使用溶解氧检测仪 ( proODO, YSI 626281 ) 测 量瓶内海水溶解氧浓度 $\left(O_{\mathrm{T}}\right.$, 精度 0.01 $\left.\mathrm{mg} \cdot \mathrm{L}^{-1}\right)$; 测完后, 采用锡管纸将试验瓶完全密 封包裹, 以进行黑暗条件下的实验。再经过约 24 $\mathrm{h}$ 后, 测量试验瓶内水体的溶解氧浓度 $\left(O_{\mathrm{T}+1}\right)$; 测 完后, 将 $\mathrm{A}$ 组实验个体置于同等光照条件下, 以 进行光照条件下的实验。以此类推, $\mathrm{A}$ 组实验共 持续 $11 \mathrm{~d}$ (6 次光照条件和 5 次黑暗条件)。

针对 B 组个体, 采用锡管纸将试验瓶完全密 封包裹, 以进行黑暗条件下的实验。密封后的试 验瓶放置约 $24 \mathrm{~h}$ 后, 使用溶解氧检测仪测量瓶内 海水溶解氧浓度 $\left(O_{\mathrm{T}}\right)$; 测完后, 将试验瓶放置在 光照强度为 $100 \mathrm{~lx}$ 的光照条件下。再经过约 $24 \mathrm{~h}$ 后, 测量试验瓶内水体的溶解氧浓度 $\left(O_{\mathrm{T}+1}\right)$ 。完 成前 $2 \mathrm{~d}$ 的实验后, $\mathrm{B}$ 组实验与 $\mathrm{A}$ 组保持相同条 件同步进行, 直至实验结束。最终, B 组实验也持 续共 $11 \mathrm{~d}$ (6 次光照条件和 5 次黑暗条件)。

上述两组实验均设置空白对照组, 即在试验 瓶中不放置磷虾, 经同时段后测定实验开始时水 体的溶解氧浓度 $\left(O^{\prime}{ }_{\mathrm{T}}\right)$ 以及结束时背景溶解氧浓 度 $\left(O^{\prime}{ }_{\mathrm{T}+1}\right)$ 。实验结束后, 将所有 9 尾磷虾样本收 集, 置于 $-20{ }^{\circ} \mathrm{C}$ 冷冻条件保存。待运回陆基实验 室后转移至 $-80{ }^{\circ} \mathrm{C}$ 超低温冰箱内保存。

\section{3 样本处理}

返回实验室后,将置于 $-80{ }^{\circ} \mathrm{C}$ 超低温冰箱的 磷虾样本取出并于常温解冻。测量解冻后的磷 虾标准体长 (total length, TL; mm) 、湿体质量 ( wet mass, MW; mg ) 和性成熟度。标准体长据 MAUCHLINE ${ }^{[23]}$ 的标准, 即由眼最前端至尾节末 端之间的长度。性成熟度的划分结合 MAKAROV 和 DENYS ${ }^{[21]}$ 与 BARGMANN ${ }^{[22]}$ 的划分标准进行 判定, 文中的标准也修改为未成体 (雌性分为 $2 \mathrm{BF} 、 2 \mathrm{CF} 、 2 \mathrm{DF} 3$ 期, 雄性分为 $2 \mathrm{AM} 、 2 \mathrm{BM} 、 2 \mathrm{CM} 3$ 期)、成体 (雄性分为 $3 \mathrm{AM} 、 3 \mathrm{BM}$ 两期, 雌性分为 $3 \mathrm{AF} 、 3 \mathrm{BF} 、 3 \mathrm{CF} 、 3 \mathrm{DF} 、 3 \mathrm{EF} 5$ 期)。本实验中的 9 尾磷虾均为未成体。测量后的磷虾个体在实验 室冷冻干燥后利用微量天平 (MITTLER TOLEDO XPR2 ) 称取其干重 (dry weight, DW ; mg)。

\section{4 数据分析}

耗氧率 (respiration rate) 指动物单位时间的 耗氧量, 可分为单位体质量耗氧率( weight specific respiration rate) 和单位个体耗氧率 (individual respiration rate $)^{[20]}$ 。因此, 每尾磷虾在实验中的 单位干重耗氧率 $R_{\mathrm{T}+1}\left[\mu \mathrm{L} \cdot(\mathrm{mg} \cdot \mathrm{h})^{-1}\right]$ 及单位 个体耗氧率 $\operatorname{Rd}_{\mathrm{T}+1}\left(\mu \mathrm{L} \cdot \mathrm{h}^{-1}\right)$ 的估算方法分别 为:

$$
\begin{array}{r}
R_{\mathrm{T}+1} \frac{\left[\left(Q_{\mathrm{T}}-Q_{\mathrm{T}+1}-\left(O_{\mathrm{T}}^{\prime}-O_{\mathrm{T}+1}^{\prime}\right] \cdot V\right.\right.}{\rho \cdot W \cdot t} \\
\operatorname{Rd}_{\mathrm{T}+1} \frac{\left[\left(Q_{\mathrm{T}}-Q_{\mathrm{T}+1}-\left(O_{\mathrm{T}}^{\prime}-O_{\mathrm{T}+1}^{\prime}\right] \cdot V\right.\right.}{\rho \cdot t}
\end{array}
$$

式 (1)、式 (2) 中, $\rho$ 为氧气密度; $V$ 为实验暂养瓶 的容积; $W$ 为实验磷虾经干燥后的体质量 $(\mathrm{mg}) ; t$ 为实验所进行的时间 $(\mathrm{h}) ; O_{\mathrm{T}}$ 为试验组开始时暂 
养瓶内海水的含氧量浓度 $\left(\mathrm{mg} \cdot \mathrm{L}^{-1}\right) ; O_{\mathrm{T}+1}$ 为试 验组结束时暂养瓶内海水的含氧量浓度 $\left(\mathrm{mg} \cdot \mathrm{L}^{-1}\right) ; O^{\prime}{ }_{\mathrm{T}}$ 为空白对照组开始时暂养瓶内 海水的含氧量浓度 $\left(\mathrm{mg} \cdot \mathrm{L}^{-1}\right) ; O^{\prime}{ }_{\mathrm{T}+1}$ 为空白对 照组结束时暂养瓶内海水的含氧量浓度 $\left(\mathrm{mg} \cdot \mathrm{L}^{-1}\right)$ 。

为了分析各尾磷虾耗氧率随时间的变化是 否存在显著性差异,本研究首先利用 Shapiro-Wilk 检验分析每尾磷虾耗氧率数据是否符合正态分 布。如符合, 则利用单因子方差分析 (ANOVA) 分析各尾磷虾耗氧率之间是否存在显著性差异; 如不符合, 则利用 Kolmogorov-Smirnov test ( K-S 检 验) 分析数据。若方差齐次, 利用 LSD 事后检验 进一步分析组内两两个体之间是否存在显著性 差异;若方差非齐次,则利用 Tamhane T2 事后检 验分析组内两两个体之间是否存在显著性差异。 显著性水平 $P=0.05$ 。统计分析利用R4.0.0进 行, 所使用的程序包包括 ggplot 2 和 MASS。模拟 单位个体呼吸率随实验天数变化时, 考虑到异常 值存在, 本研究使用了稳健线性模拟方法, 以消 除异常值对趋势的影响。

\section{2 结果与分析}

\section{1 光照与黑暗条件对耗氧率的影响}

磷虾单位个体耗氧率处于 $16.13 \sim 74.54$ $\mu \mathrm{L} \cdot \mathrm{h}^{-1}$ 之间, 平均为 $(29.460 \pm 9.343) \mu \mathrm{L} \cdot \mathrm{h}^{-1}$ (表 2), 其中黑暗条件下的磷虾单位个体耗氧率 处于 $16.13 \sim 46.55 \mu \mathrm{L} \cdot \mathrm{h}^{-1}$ 之间, 平均为 $(28.93 \pm 7.28) \mu \mathrm{L} \cdot \mathrm{h}^{-1}$, 而光照条件下的磷虾 单位个体耗氧率处于 $17.76 \sim 74.54 \mu \mathrm{L} \cdot \mathrm{h}^{-1}$ 之 间, 平均为 $(29.89 \pm 10.83) \mu \mathrm{L} \cdot \mathrm{h}^{-1}$ 。对于实验
中磷虾单位体质量耗氧率, 所有个体处于 $0.1026 \sim 0.5575 \mu \mathrm{L} \cdot(\mathrm{mg} \cdot \mathrm{h})^{-1}$ 之间, 平均为 (0.269 $0 \pm 0.0784) \mu \mathrm{L} \cdot(\mathrm{mg} \cdot \mathrm{h})^{-1}$ (表 3$)$; 其 中黑暗条件下的磷虾单位体质量耗氧率处于 $0.1026 \sim 0.5575 \mu \mathrm{L} \cdot(\mathrm{mg} \cdot \mathrm{h})^{-1}$ 之间, 平均为 $(0.2673 \pm 0.0840) \mu \mathrm{L} \cdot(\mathrm{mg} \cdot \mathrm{h})^{-1}$, 而光照条 件下的磷虾单位体质量耗氧率处于 0.1286 $0.4664 \mu \mathrm{L} \cdot(\mathrm{mg} \cdot \mathrm{h})^{-1}$ 之间, 平均为 $(0.2705 \pm 0.0742) \mu \mathrm{L} \cdot(\mathrm{mg} \cdot \mathrm{h})^{-1}$ 。

从整体上来讲, 磷虾单位体质量耗氧率与体 长之间无相关性 $\left(R^{2}=0.176, n=9, P=0.261\right)$ 。 就单位个体耗氧率而言, 随着实验天数的增加, $\mathrm{A}$ 组磷虾的个体耗氧率先下降再保持稳定, 而 $\mathrm{B}$ 组 磷虾的个体耗氧率呈增加趋势 (图 2)。

\section{2 两组磷虾耗氧率的个体差异}

整体上来看, $\mathrm{A}$ 组磷虾单位个体耗氧率变化 较 B 组更为显著 (图 3)。A 组中实验个体 2 在死 亡前的单位个体耗氧率出现剧烈的波动。 $\mathrm{A}$ 组 中, ANOVA 结果显示, 个体 $1 、 3$ 和 4 的单位个体 耗氧率存在显著性差异 $(F=25.478, \mathrm{df}=32$, $P<0.001)$; LSD 检验也显示, 两两个体间也存在 着显著性差异 (个体 1 与个体 $3: P<0.001$; 个体 1 与个体 $4: P<0.001$; 个体 3 与个体 $4: P=0.022$ $<0.05) 。 B$ 组中, ANOVA 结果显示, 4 个实验个 体的单位个体耗氧率之间存在显著性差异 $(F=$ 14.090, df = 43, $P<0.001$ ); Tamhane T2 事后检 验显示, 两两个体间并非均存在显著性差异, 实 验个体 5 和 $8(P=0.865) 、 6$ 和 $7(P=0.839)$ 以 及 7 与 $8(P=0.119)$ 之间并无显著性差异 (表 4)。

表 2 南极大磷虾单位个体耗氧率的平均值、最小值和最大值

Tab.2 Average, minimum and maximum values of individual respiration rate of Euphausia superba

\begin{tabular}{cccc}
\hline $\begin{array}{c}\text { 磷虾编号 } \\
\text { Krill serial }\end{array}$ & $\begin{array}{c}\text { 实验中平均耗氧率 } \\
/\left(\mu \mathrm{L} \cdot \mathrm{h}^{-1}\right) \\
\text { Average respiration rate }\end{array}$ & $\begin{array}{c}\text { 耗氧率最小值 } \\
/\left(\mu \mathrm{L} \cdot \mathrm{h}^{-1}\right) \\
\text { Min. respiration rate }\end{array}$ & $\begin{array}{c}\text { 耗氧率最大值 } \\
\left(\mu \mathrm{L} \cdot \mathrm{h}^{-1}\right) \\
\text { Max. respiration rate }\end{array}$ \\
\hline 1 & $33.860 \pm 5.828$ & 25.20 & 45.25 \\
2 & $59.920 \pm 16.330$ & 45.03 & 74.54 \\
3 & $21.190 \pm 2.444$ & 16.13 & 24.04 \\
4 & $25.870 \pm 2.630$ & 23.42 & 31.85 \\
5 & $25.080 \pm 3.805$ & 21.96 & 32.59 \\
6 & $33.540 \pm 2.759$ & 28.93 & 39.78 \\
7 & $31.760 \pm 3.680$ & 25.99 & 35.51 \\
8 & $23.920 \pm 5.746$ & 17.76 & 31.39 \\
9 & $29.270 \pm 2.264$ & 25.75 & 74.54 \\
\hline
\end{tabular}


表 3 南极大磷虾单位体质量耗氧率的平均值、最小值和最大值

Tab.3 Average, minimum and maximum values of weight specific respiration rate of Euphausia superba

\begin{tabular}{|c|c|c|c|}
\hline $\begin{array}{l}\text { 磷虾编号 } \\
\text { Krill serial }\end{array}$ & $\begin{array}{l}\text { 实验中平均耗氧率 } \\
/\left[\mu \mathrm{L} \cdot(\mathrm{mg} \cdot \mathrm{h})^{-1}\right] \\
\text { Average respiration rate }\end{array}$ & $\begin{array}{c}\text { 耗氧率最小值 } \\
/\left[\mu \mathrm{L} \cdot(\mathrm{mg} \cdot \mathrm{h})^{-1}\right] \\
\text { Min. respiration rate }\end{array}$ & $\begin{array}{c}\text { 耗氧率最大值 } \\
/\left[\mu \mathrm{L} \cdot(\mathrm{mg} \cdot \mathrm{h})^{-1}\right] \\
\text { Max. respiration rate }\end{array}$ \\
\hline 1 & $0.2844 \pm 0.0489$ & 0.2116 & 0.3800 \\
\hline 2 & $0.3078 \pm 0.0839$ & 0.2313 & 0.3828 \\
\hline 3 & $0.1348 \pm 0.0155$ & 0.1026 & 0.1529 \\
\hline 4 & $0.3132 \pm 0.0391$ & 0.2835 & 0.3856 \\
\hline 5 & $0.2575 \pm 0.0318$ & 0.2254 & 0.3346 \\
\hline 6 & $0.2445 \pm 0.0201$ & 0.2108 & 0.2899 \\
\hline 7 & $0.2710 \pm 0.3140$ & 0.2218 & 0.3165 \\
\hline 8 & $0.3756 \pm 0.0902$ & 0.2788 & 0.5575 \\
\hline 9 & $0.2504 \pm 0.0194$ & 0.2203 & 0.2685 \\
\hline 全部 All & $0.2690 \pm 0.0784$ & 0.1026 & 0.5575 \\
\hline
\end{tabular}

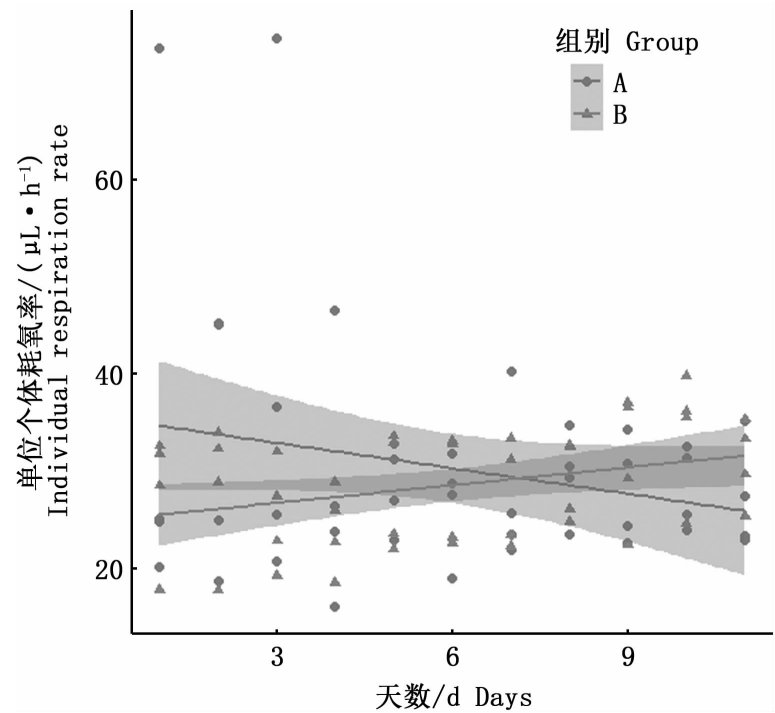

图 2 各组南极大磷虾单位个体耗氧率随天数的变化

Fig. 2 Variations in individual respiration rate of Euphausia superba with days in two groups 注:阴影部分为 $95 \%$ 置信区间

Note: The shaded areas signify $95 \%$ confidence intervals

如仅考虑同等实验条件,从加人 9 号个体后 开始, ANOVA 结果显示, 8 组实验磷虾 (除去 2 号 虾) 的单位个体耗氧率仍存在显著性差异 $(F=$ $20.134, \mathrm{df}=48, P<0.001)$, 但 Tamhane T2 事后 检验显示, $A$ 组的个体 $1 、 3$ 和 4 之间的单位个体 耗氧率存在显著性差异, 而 $B$ 组中的个体 $6 、 7$ 以 及 8 之间的单位个体耗氧率则不存在显著性差 异。

\section{3 讨论}

本次实验基于海上暂养实验完成了现场光 照条件对磷虾冬季耗氧率的影响研究。在此之 前, 针对冬季磷虾耗氧率的研究极为有限 (表 5),
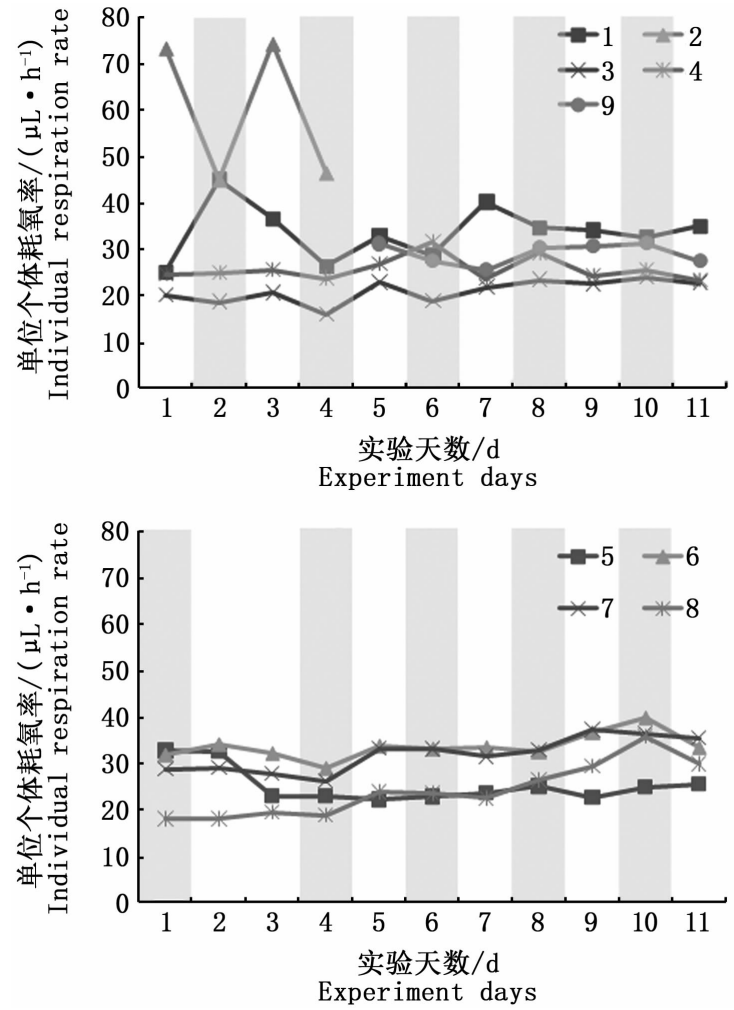

图 3 光照 (黑暗) 条件下南极大磷虾 单位个体耗氧率变化

Fig. 3 Variations in individual respiration rate of Euphausia superba

注: 灰色阴影部分表示黑暗条件

Note: The grey shades signify dark regimes

且仅有的研究多在陆基实验室内完成。为了尽 可能地模拟实际情况, 本研究模拟了磷虾自然条 件下的生理状态, 实验所使用的密闭瓶浸没于当 地循环海水中, 以保证实验环境水温与当地海水 完全一致,这在一定程度上可以保证实验环境更 接近磷虾生活的自然环境。 
表 4 B 组实验磷虾单位个体耗氧率两两差异的 Tamhane T2 事后检验

Tab. 4 Tamhane T2 post hoc test for examining individual respiration rate of Euphausia superba in group B

\begin{tabular}{|c|c|c|c|c|c|c|c|c|}
\hline \multirow{2}{*}{$\begin{array}{c}\text { 组别 } \\
\text { Group }\end{array}$} & \multicolumn{4}{|c|}{ 标准误差 Standard error } & \multicolumn{4}{|c|}{$P$ 值 $P$ value } \\
\hline & 5 & 6 & 7 & 8 & 5 & 6 & 7 & 8 \\
\hline 5 & - & 0.91871 & 1.58693 & 1.70702 & - & - & - & - \\
\hline 6 & - & - & 1.75490 & 1.86420 & 0.000 & - & - & - \\
\hline 7 & - & - & - & 2.26926 & 0.002 & 0.839 & - & - \\
\hline 8 & - & - & - & - & 0.865 & 0.006 & 0.119 & - \\
\hline
\end{tabular}

表 5 光照影响磷虾耗氧率研究

Tab. 5 Effect of light on respiration rate of Euphausia superba

\begin{tabular}{|c|c|c|c|c|c|}
\hline $\begin{array}{c}\text { 来源 } \\
\text { Researcher }\end{array}$ & $\begin{array}{c}\text { 实验地点 } \\
\text { Experiment } \\
\text { location }\end{array}$ & $\begin{array}{c}\text { 实验时间 } \\
\text { Experiment } \\
\text { time }\end{array}$ & $\begin{array}{c}\text { 条件 } \\
\text { Condition }\end{array}$ & $\begin{array}{c}\text { 单位个体耗氧率 } \\
/\left(\mu \mathrm{L} \cdot \mathrm{h}^{-1}\right) \\
\text { Individual } \\
\text { respiration rate }\end{array}$ & $\begin{array}{c}\text { 单位体质量耗氧率 } \\
/\left[\mu \mathrm{L} \cdot(\mathrm{mg} \cdot \mathrm{h})^{-1}\right] \\
\text { Weight specific respiration rate }\end{array}$ \\
\hline $\mathrm{HIRCHE}^{[24]}$ & 海上 & 夏季 & 黑暗 & & $0.63 \sim 0.88$ \\
\hline SWADLING 等 ${ }^{[25]}$ & 陆基实验室 & 秋季 & $\begin{array}{c}12: 12^{\mathrm{a}} \\
\text { 水流 } 3 \mathrm{~cm} \cdot \mathrm{s}^{-1}\end{array}$ & 25.17 & \\
\hline \multirow[t]{4}{*}{ BROWN 等 $[12]$} & 陆基实验室 & 冬季 & 黑暗 & & 0.35 \\
\hline & & & 自然光照 & & 0.22 \\
\hline & & 夏初 & 黑暗 & & 0.56 \\
\hline & & & 自然光照 & & 0.46 \\
\hline \multirow[t]{3}{*}{ TESCHKE 等 $[11]$} & 陆基实验室 & 秋冬季 & 光照 ${ }^{\mathrm{b}}$ & $(22.9 \pm 8.8) \sim(53.7 \pm 12.9)$ & \\
\hline & & & 黑暗 & $(22.0 \pm 3.1) \sim(30.0 \pm 8.4)$ & \\
\hline & & & $12: 12^{b}$ & $(26.6 \pm 7.3) \sim(44.7 \pm 11.1)$ & \\
\hline \multirow[t]{2}{*}{ 本文 } & 海上 & 冬季 & 黑暗 & $28.67 \pm 7.49$ & $0.2673 \pm 0.0830$ \\
\hline & & & 光照 ${ }^{\mathrm{c}}$ & $30.12 \pm 10.68$ & $0.2705 \pm 0.0734$ \\
\hline
\end{tabular}

注: ${ }^{\mathrm{a}}$ 指实验过程中黑暗与光照各 $12 \mathrm{~h}$;

${ }^{b}$ 光照指实验中光照强度为 $200 \mathrm{~lx} ; 12: 12$ 指光照与黑暗各 $12 \mathrm{~h}$, 其中光照强度为 $50 \mathrm{~lx}$;

${ }^{c}$ 实验的光照强度为 $100 \mathrm{~lx}$

Note: ${ }^{\text {a }}$ The darkness and light conditions during the experiment are for 12 hours each;

b The light intensity of light condition is $200 \mathrm{~lx} ; 12: 12$ means the darkness and light conditions during the experiment for 12 hours each, the light intensity of light condition is $50 \mathrm{~lx}$;

${ }^{c}$ The light intensity of experiment is $100 \mathrm{~lx}$

\section{1 磷虾冬季耗氧率}

高密度的磷虾分布海域位于南极大西洋扇 区的威德尔海、南奥克尼群岛、南设得兰群岛周 围以及南桑维奇群岛西部水域 ${ }^{[26]}$, 这些水域的水 温、饵料、海冰覆盖情况及光周期和光强度 ${ }^{[27]}$ 均 存在着周期性的年际变化。环境特征的强烈季 节性变化意味着磷虾的生长与发育也呈现出相 应的季节性特征。但作为南大洋生态系统中的 关键种, 磷虾已进化出相应的生命周期以适应这 种高度季节性的环境。冬季, 磷虾通过降低代谢
速率、缩小体型以及改变摄食策略等方法,由夏 季的高代谢、高消耗生存模式向冬季的低代谢、 低消耗模式转化, 这种季节性生命周期变化使得 磷虾能够在一年中较好地分配新陈代谢能力, 即 在食物源丰富且自然环境有利的夏季进行消耗 较大的繁殖过程, 而在条件极为苛刻的冬季转换 为节能模式 ${ }^{[28]}$ 。耗氧率作为衡量代谢率的一个 重要指标,其变化可以较好地反映磷虾体内新陈 代谢水平。

本研究结果显示, 冬季黑暗条件下磷虾的单 
位体质量耗氧率平均为 $(0.2673 \pm 0.0840) \mu \mathrm{L}$. $(\mathrm{mg} \cdot \mathrm{h})^{-1}$, 这明显低于夏季黑暗条件下的单位体 质量耗氧率 $0.63 \sim 0.88 \mu \mathrm{L} \cdot(\mathrm{mg} \cdot \mathrm{h})^{-1[24]}$, 也 低于实验室模拟的冬季黑暗条件下的单位体质 量耗氧率 $0.35 \mu \mathrm{L} \cdot(\mathrm{mg} \cdot \mathrm{h})^{-1}$ 和夏初黑暗条件 下的单位体质量耗氧率 $0.56 \mu \mathrm{L} \cdot(\mathrm{mg}$.

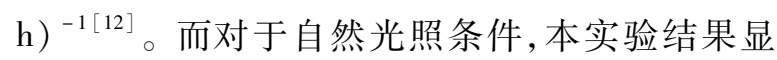
示, 磷虾的单位体质量耗氧率平均为 $(0.2704 \pm$ $0.0740) \mu \mathrm{L} \cdot(\mathrm{mg} \cdot \mathrm{h})^{-1}$, 这略高于实验室模拟 的冬季自然光照条件下的单位体质量耗氧率 $0.22 \mu \mathrm{L} \cdot(\mathrm{mg} \cdot \mathrm{h})^{-1}$, 但却低于夏初黑暗条件下 的单位体质量耗氧率 $0.46 \mu \mathrm{L} \cdot(\mathrm{mg} \cdot \mathrm{h})^{-1[12]}$ 。 针对单位个体呼吸率, 本研究结果与 SWADLING 等 $^{[25]}$ 类似。由此, 可以初步判断, 冬季磷虾的单 位体质量耗氧率较夏季低, 也进一步说明冬季磷 虾的新陈代谢率有所下降 ${ }^{[29]}$ 。但需要注意的是, 实验室模拟条件下所获得的相关数据与自然条 件下仍有所差别, 造成该结果的可能原因之一在 于磷虾的环境适应能力。经过长期进化, 磷虾已 适应并反馈南极特有的季节性光周期变化。正 因为如此, 通常在南极现场开展磷虾生长率等方 面的实验时, 仅设定较短的时间尺度 (如 $4 \mathrm{~d})^{[5]}$, 以防止磷虾因适应新的环境而在生理上做出改 变。

\section{2 光照对冬季磷虾耗氧率的影响}

磷虾耗氧率存在较为显著的季节性变化 (表 5 ), 而引起这种季节性变化的机制仍未明确。 ATKINSON 和 SNYDER ${ }^{[30]}$ 就食物是否影响不同 季节磷虾代谢速率开展了研究, 通过将夏季磷虾 分组暂养并以食物浓度作为条件变量检验两组 磷虾耗氧率的差异, 结果显示处于高浓度食物环 境的磷虾耗氧率和清除率迅速上升。但针对秋 季的实验却得到了不同的结果 ${ }^{[31]}$, 后者将磷虾放 置于食物充足的环境 $11 \mathrm{~d}$, 耗氧率并未发生显著 升高, 且耗氧率仅为夏季的三分之一。这两个实 验在一定程度上说明食物浓度并非导致磷虾代 谢率变化的主要原因。

南大洋日照时长具有明显的季节性差异, 在 磷虾的主要分布纬度 $\left(50 \sim 60^{\circ} \mathrm{S}\right)$, 日照时长由 12 月的 $20 \mathrm{~h}$ 转变至 6 月的 $6 \mathrm{~h}$, 这被认为可能是调 节磷虾代谢状态的环境信号。TESCHKE 等 ${ }^{[11]}$ 发 现, 4-7 月磷虾的耗氧率的确受到了光照条件的 影响, 处于光照条件下的磷虾耗氧率明显高于处
于黑暗条件下的磷虾。这表明光照强度高时, 磷 虾耗氧率也会升高, 并由此推断光照强度及其持 续时间的年度过程可为控制磷虾的生理过程提 供可靠且可预测的信息。这与 KAWAGUCHI 等 ${ }^{[32]}$ 的研究结论相同, 其认为在低水平的食物条 件下, 磷虾耗氧率的增加与太阳辐射增加有关。 然而, BROWN 等 ${ }^{[12]}$ 的实验却得到了相反的结 论, 即黑暗条件下的磷虾耗氧率要高于光照条 件,而该文作者分析认为实验水箱过大 $(100 \mathrm{~L}$ 、 $600 \mathrm{~L} 、 1600 \mathrm{~L})$ 是造成这种差异的原因, 即冬季 磷虾因已适应黑暗环境而增加游泳活动, 其耗氧 率也因活动量增加而高于夏季 (光照组) 磷虾。 由此, 为了降低因游泳活动增加而带来的影响, 本研究使用了体积较小的实验瓶 $(500 \mathrm{~mL}$ ) 作为 容器, 这减少了游泳行为对耗氧率结果带来的影 响, 但结果并未显示出黑暗与光照条件下的磷虾 耗氧率存在较为显著的差异。本研究中, 在接近 一个自然日的周期内, 通过黑暗和 $100 \mathrm{~lx}$ 光强两 种条件的变化, 对试验组磷虾进行反复实验, 结 果表明这种条件的改变对磷虾的耗氧率影响并 不显著, 除 2 号样本外 (可能因捕捞过程对个体 造成的影响), 磷虾的耗氧率水平始终未发生较 明显的波动。这在一定程度上说明, 冬季时期, 磷虾的呼吸速率已降到一个较低的水平, 日变化 尺度上的光照条件变化并不会对它的新陈代谢 速率产生显著影响, 进一步表明磷虾低代谢模式 可能具有一定的稳定性。考虑到本实验的样本 较少, 今后可进一步增加样本证实这种机制假设 的可靠性。此外, 本次实验还发现, 黑暗组磷虾 的耗氧率变化 (标准差) 低于光照组, 表明光照条 件下磷虾的耗氧率波动更大, TESCHKE 等 ${ }^{[11]}$ 的 实验中也发现了类似现象, 且光照时间越长, 光 照强度越大, 这种波动也越强。这侧面说明了冬 季时磷虾低代谢模式较稳定, 而本实验中提供的 光照条件在一定程度上影响了这种稳定, 从而使 得磷虾的耗氧率出现相应的波动。

总体上来看, 影响磷虾耗氧率的因素较多, 有生物因素和非生物因素, 生物因素有个体大 小、饥饿与摄食、活动与应激及生物周期, 非生物 因素有温度、盐度、氧张力 $\left(\mathrm{PO}_{2}\right)$ 及 $\mathrm{pH}$ 等 ${ }^{[20]}$ 。由 于海上条件所限, 较难对各项实验条件均做到精 确控制。在实验处理上仍需进一步完善, 如实验 中所用海水为当地自然循环海水, 温度不易控 
制。为此,为防止对实验结果产生影响, 今后的 实验中应加以改善, 尽可能地稳定实验条件, 减 少误差。为了深人研究光照条件对磷虾代谢模 式的影响, 今后可从光强、光照时长等因素进行 设置,并在不同季节展开相应的现场实验, 以期 全面地揭示磷虾耗氧率季节性变化的机制。

致谢: 感谢中国水产总公司 “龙腾”轮船长、政委、 各位船员实验过程中给予的帮助。本项目还得 到了农业农村部南极海洋生物资源开发利用项 目的支持。

\section{参考文献:}

[1] EVERSON I. Krill: Biology, ecology and fisheries [M]. Hamburg: Springer, 2000.

[2 ] SIEGEL V, LOEB V. Recruitment of Antarctic krill Euphausia superba and possible causes for its variability $[\mathrm{J}]$. Marine Ecology Progress Series, $1995,123(1-3): 45-56$.

[3] HAGEN W, KATTNER G, TERBRÜGGEN A, et al. Lipid metabolism of the Antarctic krill Euphausia superba and its ecological implications $[\mathrm{J}]$. Marine Biology, 2001, 139(1) : 95 - 104.

[4] KAWAGUCHI K, ISHIKAWA S, MATSUDA 0. The overwintering strategy of Antarctic krill (Euphausia superba Dana) under the coastal fast ice off the Ongul Islands in Lutzow-Holm Bay, Antarctica $[\mathrm{J}]$. Memoirs of National Institute of Polar Research, 1986, 44(SI) : $67-85$.

[5] LANGDON B Q, ROBIN M R. Behavioral and physiological characteristics of the Antarctic krill, Euphausia superba $[\mathrm{J}]$. American Zoologist, 1991, $31(1): 49-63$.

[6] TORRES J J, DONNELLY J, HOPKINS T L, et al. Proximate composition and overwintering strategies of Antarctic micronektonic crustacea $[\mathrm{J}]$. Marine Ecology Progress Series, 1994, 113 (3): 221 232 .

[7] IKEDA T, DIXON P. Body shrinkage as a possible over-wintering mechanism of the Antarctic krill, Euphausia superba Dana [ J ]. Journal of Experimental Marine Biology and Ecology, 1982, 62 (2) : $143-151$.

[8] MARSCHALL H P. The overwintering strategy of Antarctic krill under the pack-ice of the Weddell Sea [J]. Polar Biology, 1988, 9(2) : 129 - 135.
[9] MEYER B, SABOROWSKI R, ATKINSON A, et al. Seasonal differences in citrate synthase and digestive activity in larval and postlarval Antarctic krill, Euphausia superba [ J ]. Marine Biology (2002), 141: $855-862$.

[10 ] CULLEN M, KAUFMANN R S, LOWERY M S. Seasonal variation in biochemical indicators of physiological status in Euphausia superba from Port Foster, Deception Island, Antarctica[J]. Deep Sea Research Part II: Topical Studies in Oceanography, $2003,50(10): 1787-1798$.

[11] TESCHKE M, KAWAGUCHI S, MEYER B. Simulated light regimes affect feeding and metabolism of Antarctic krill, Euphausia superba $[\mathrm{J}]$. Limnology and Oceanography, 2007, 52(3) : 1046 - 1054.

[12] BROWN M, KAWAGUCHI S, CANDY S, et al. Long-term effect of photoperiod, temperature and feeding regimes on the respiration rates of Antarctic krill (Euphausia superba) [J]. Open Journal of Marine Science, 2013, 3: 40 - 51 .

[13] 徐鹏翔, 李莹春, 朱国平, 等. 光照条件下南极磷 虾的行为观察 $[\mathrm{J}]$. 水产学报, 2012, 36(2) : 300 -305 .

XU P X, LI Y C, ZHU G P, et al. Observation on behaviours of Antarctic krill (Euphausia superba) in lighting condition $[\mathrm{J}]$. Journal of Fisheries of China, 2012, 36(2) : 300-305.

[14] 杨文杰, 朱国平, 胡桂森, 等. 南极磷虾的适温效 应试验 $[\mathrm{J}]$. 江苏农业科学, 2015, 43(2) : $235-$ 238.

YANG W J, ZHU G P, HU G S, et al. Experiment on optimum temperature effect of Antarctic krill $[\mathrm{J}]$. Jiangsu Agricultural Sciences, 2015, 43(2) : 235 238.

[15] ZHU G P, LIU Z J, YANG Y, et al. Thermal and saline tolerance of Antarctic krill Euphausia superba under controlled in-situ aquarium conditions $[\mathrm{J}]$. Journal of Oceanology and Limnology, 2019, 37 (3) : $1080-1089$.

[16] 李莹春, 朱国平, 孟 涛, 等. 人工条件下南极磷 虾的温度耐受性试验观察 $[\mathrm{J}]$. 江苏农业科学, 2012, 40(9) : $204-206$.

LI Y C, ZHU G P, MENG T, et al. Experimental observation on temperature tolerance of Antarctic krill under artificial conditions $[\mathrm{J}]$. Jiangsu Agricultural Sciences, 2012, 40(9) : $204-206$.

[17] 胡士国, 许柳雄, 王 腾, 等. 2017 年秋季南奥 克尼群岛海域南极大磷虾垂直分布与光照及垂向 
温盐关系 $[\mathrm{J}]$. 海洋渔业, 2019, 41 (2) : 160 168.

HU S G, XU L X, WANG T, et al. Vertical distribution of Euphausia superba in sea areas around the South Orkney Islands in autumn 2017 and its relations with illumination, vertical temperature and salinity $[\mathrm{J}]$. Marine Fisheries, 2019, $41(2)$ : 160 168.

[18］朱国平, 王 芮, 朱小艳, 等. 2013 年冬季南乔 治亚岛南极磷虾群昼夜垂直移动研究 $[\mathrm{J}]$. 水产 学报, 2015, 39(8): $1242-1249$.

ZHU G P, WANG R, ZHU X Y, et al. Diel vertical migration of Antarctic krill aggregation in South Georgia Island in the austral winter season of 2013 [J]. Journal of Fisheries of China, 2015, 39 (8) : $1242-1249$.

[19］朱国平, 杨 洋, 王 苪, 等. 采用渔业声学方法 研究 2016 年秋季布兰斯菲尔德海峡南极磷虾群 昼夜垂直移动特征及其影响因素 $[\mathrm{J}]$. 水产学报, 2018, 42(10) : 1541 - 1549.

ZHU G P, YANG Y, WANG R, et al. Diel vertical migration of Antarctic krill (Euphausia superba) swarm in the Bransfield Strait during austral autumn 2016 inferred from acoustic data and the relations to environmental factors $[\mathrm{J}]$. Journal of Fisheries of China, 2018, 42(10): $1541-1549$.

[20] 林小涛, 张秋明, 许忠能, 等. 虾蟹类呼吸代谢研 究进展 $[\mathrm{J}]$. 水产学报, 2000, 24(6) : 575-580. LIN $\mathrm{X}$ T, ZHANG Q M, XU Z N, et al. Advancement of the study on respiratory metabolism of Decapod crustaceans $[\mathrm{J}]$. Journal of fisheries of China, 2000, 24(6): 575 - 580 .

[21] MAKAROV R R, DENYS C J. Stages of sexual maturity of Euphausia superba Dana[R]. BIOMASS Handbook Series, 1981(11): 1 - 13.

[22] BARGMANN H E. The development and life-history of adolescent and adult krill, Euphausia superba [R]. Discovery Report, 1945(23): 103 - 176.

[23 ] MAUCHLINE J. Measurement of body length of Euphausia superba Dana $[\mathrm{R}]$. // ANON. Biomass
Handbook. Cambridge: Scientific Committee on Antarctic Research (SCAR), 1980(4): 1 - 9.

[24] HIRCHE H J. Excretion and respiration of the Antarctic krill Euphausia superba [ J ]. Polar Biology, 1983, 1(4) : $205-209$.

[25] SWADLING K, RITZ D, NICOL S, et al. Respiration rate and cost of swimming for Antarctic krill, Euphausia superba, in large groups in the laboratory [J]. Marine Biology, 2005, 146 (6): 1169 1175 .

[26] 黄洪亮, 陈雪忠, 冯春雷. 南极磷虾资源开发现 状分析 [J]. 渔业现代化, 2007(1) : 48-51.

HUANG H L, CHEN X Z, FENG C L. Exploitation status of Antarctic krill resource $[\mathrm{J}]$. Fisheries Modernization, 2007 (1) : $48-51$.

[27] KNOX A G. The biology of the Southern Ocean [M]. Cambridge: University Press, 1994.

[28 ] TESCHKE M, KAWAGUCHI S, MEYER B. Effects of simulated light regimes on maturity and body composition of Antarctic krill, Euphausia superba [J]. Marine Biology, 2008, 154(2) : 315 - 324.

[29] AUERSWALD L, MEYER B, TESCHKE M, et al. Physiological response of adult Antarctic krill, Euphausia superba, to long-term starvation $[\mathrm{J}]$. Polar Biology, 2015, 38(6) : 763 - 780.

[30 ] ATKINSON A, SNYDER R. Krill-copepod interactions at South Georgia, Antarctica, I. Omnivory by Euphausia superba $[\mathrm{J}]$. Marine Ecology Progress Series, 1997, 160(8): $63-76$.

[31] ATKINSON A, MEYER B, STUVBING D, et al. Feeding and energy budgets of Antarctic krill Euphausia superba at the onset of winter-II. Juveniles and adults [ $\mathrm{J}]$. Limnology and Oceanography, 2002, 47 (4) : $953-966$.

[32] KAWAGUCHI S, KING R, MEIJERS R, et al. An experimental aquarium for observing the schooling behaviour of Antarctic krill (Euphausia superba) [J]. Deep-Sea Research Part II: Topical Studies in Oceanography, 2010, $57(7-8): 683-692$. 


\title{
Effect of in-situ simulated light levels on respiration rate of Euphausia superba in winter
}

\author{
HU Guangzhao ${ }^{1,2}$, LIU Zijun ${ }^{1,2}$, ZHU Guoping ${ }^{1,2,3,4}$ \\ (1. College of Marine Sciences, Shanghai Ocean University, Shanghai 201306, China; 2. Center for Polar Research, \\ Shanghai Ocean University, Shanghai 201306, China; 3. Polar Marine Ecosystem Lab, Key Laboratory of Sustainable \\ Exploitation of Oceanic Fisheries Resources, Ministry of Education, Shanghai Ocean University, Shanghai 201306, China;
}

4. National Engineering Research Center for Oceanic Fisheries, Shanghai 201306, China)

\begin{abstract}
As the key species in the Southern Ocean, how Antarctic krill (Euphausia superba, hereafter krill) survives through the extremely severe winter time is always one of the key issues for krill ecology. Krill can utilize different strategies to tackle the long winter time, and in which the most effective pattern is to decrease the metabolism and subsequently to reduce the energy consumption. The studies available indicate that the lighting condition could be the key of low energy consumption pattern; however, the in situ data are extremely limited. The oxygen consumption rate usually reflects the metabolic rate of organisms. Therefore, in order to explore the effect of in situ lighting conditions on the metabolism of krill, this study measured the oxygen consumption of krill during the successive 11 days under dark and lighting conditions based on the in situ aquarium experiment. In the experiment, the krill was placed in a closed bottle, and the respiration rate of the krill over a period of time was obtained by measuring the difference in the oxygen concentration of the seawater in the bottle before and after the experiment. A robust linear simulation method was used to eliminate the influence of outliers on the trend of daily variation in respiration rate of krill. Results showed that the average weight specific respiration rate of krill under light conditions was $\left(\begin{array}{llllll}0.270 & 4 \pm 0.074 & 0\end{array}\right)$ $\mu \mathrm{L} \cdot(\mathrm{mg} \cdot \mathrm{h})^{-1}$, and the average weight specific respiration rate under dark conditions was $(0.2673 \pm$ $0.0840) \mu \mathrm{L} \cdot \mathrm{mg}(\cdot \mathrm{h})^{-1}$. In this study, in a period close to a natural day, the experimental group of krill was repeatedly tested by changing the conditions of darkness and $100 \mathrm{~lx}$ light intensity. The experimental results showed that the oxygen consumption level of krill did not show significant fluctuation. The comparative analysis demonstrated that the oxygen consumption of krill in the winter was significantly lower than that in the summer. The change of lighting condition within a natural day did not impact significantly on oxygen consumption of krill, but the stability of respiration rate of krill was impacted. The study supported the observation that krill was on low metabolism pattern during the winter time, and the stability of such pattern was also proved. The conclusion in the study will provide important basic data for revealing the overwintering mechanism of krill.
\end{abstract}

Keywords: Euphausia superba; respiration rate; overwintering; metabolism 